\title{
Discrete dipole approximation simulations of gold nanorod optical properties : choice of input parameters and comparison with experiment
}

\author{
Citation for published version (APA): \\ Ungureanu, C., Rayavarapu, R. G., Manohar, S., \& Leeuwen, van, T. G. (2009). Discrete dipole approximation \\ simulations of gold nanorod optical properties : choice of input parameters and comparison with experiment. \\ Journal of Applied Physics, 105(10), 102032-1/7. [102032]. https://doi.org/10.1063/1.3116139
}

DOI:

10.1063/1.3116139

Document status and date:

Published: 01/01/2009

\section{Document Version:}

Publisher's PDF, also known as Version of Record (includes final page, issue and volume numbers)

\section{Please check the document version of this publication:}

- A submitted manuscript is the version of the article upon submission and before peer-review. There can be important differences between the submitted version and the official published version of record. People interested in the research are advised to contact the author for the final version of the publication, or visit the DOI to the publisher's website.

- The final author version and the galley proof are versions of the publication after peer review.

- The final published version features the final layout of the paper including the volume, issue and page numbers.

Link to publication

\footnotetext{
General rights

- You may freely distribute the URL identifying the publication in the public portal. follow below link for the End User Agreement:

www.tue.nl/taverne

\section{Take down policy}

If you believe that this document breaches copyright please contact us at:

openaccess@tue.nl

providing details and we will investigate your claim.
}

Copyright and moral rights for the publications made accessible in the public portal are retained by the authors and/or other copyright owners and it is a condition of accessing publications that users recognise and abide by the legal requirements associated with these rights.

- Users may download and print one copy of any publication from the public portal for the purpose of private study or research.

- You may not further distribute the material or use it for any profit-making activity or commercial gain

If the publication is distributed under the terms of Article $25 \mathrm{fa}$ of the Dutch Copyright Act, indicated by the "Taverne" license above, please 


\title{
Discrete dipole approximation simulations of gold nanorod optical properties: Choice of input parameters and comparison with experiment
}

\author{
Constantin Ungureanu, ${ }^{1, a)}$ Raja Gopal Rayavarapu, ${ }^{1}$ Srirang Manohar, ${ }^{1, b)}$ and \\ Ton G. van Leeuwen ${ }^{1,2}$ \\ ${ }^{1}$ Biophysical Engineering Group, Faculty of Science and Technology, Institute for Biomedical Technology \\ (BMTI), University of Twente, PB 217, 7500AE Enschede, The Netherlands \\ ${ }^{2}$ Laser Center, Academic Medical Center, University of Amsterdam, PB 22700, 1100DE Amsterdam, \\ The Netherlands
}

(Received 26 March 2008; accepted 13 August 2008; published online 19 May 2009)

Gold nanorods have interesting optical properties due to surface plasmon resonance effects. A variety of biomedical applications of these particles have been envisaged and feasibilities demonstrated in imaging, sensing, and therapy based on the interactions of light with these particles. In order to correctly interpret experimental data and tailor the nanorods and their environments for optimal use in these applications, simulations of the optical properties of the particles under various conditions are essential. Of various numerical methods available, the discrete dipole approximation (DDA) approach implemented in the publicly available DDSCAT code is a powerful method that had proved popular for studying gold nanorods. However, there is as yet no universal agreement on the shape used to represent the nanorods and on the dielectric function of gold required for the simulations. We systematically study the influence of these parameters on simulated results. We find large variations in the position of plasmon resonance peaks, their amplitudes, and shapes of the spectra depending on the choice of the parameters. We discuss these in the light of experimental optical extinction spectra of gold nanorods synthesized in our laboratory. We show that much care should be taken and prudence applied before DDA results be used to interpret experimental data and to help characterize nanoparticles synthesized. () 2009 American Institute of Physics.

[DOI: $10.1063 / 1.3116139]$

\section{INTRODUCTION}

Gold nanoparticles exhibit striking optical properties due to the phenomenon of surface plasmon resonance. ${ }^{1}$ Conduction electrons are set into resonant oscillation at certain wavelengths of incident light, which is manifested in a peaking in the interactions between photons and the nanoparticles. In general, the wavelengths at which the plasmon peaks occur are dependent not only on size and shape but also coupling between particles and properties of the embedding medium. In the case of spherical gold nanoparticles with diameters of $<20 \mathrm{~nm}$ dispersed in water, the scattering and absorption spectra show sharp and narrow peaks at around $520 \mathrm{~nm}$. Due to their asymmetry, gold nanorods (GNRs) show two plasmon resonances: a longitudinal mode and a transverse mode due to electron oscillations along the major and minor axes of the particles, respectively. The transverse plasmon peak remains in the vicinity of $520 \mathrm{~nm}$ but the longitudinal plasmon (LP) resonance peak can be tuned to occur in the visible and the near-infrared (NIR) wavelengths by changing, for example, the aspect ratio of the particles. In addition to maxima in the scattering and absorption, luminescence effects have also been observed. ${ }^{2}$ Further, certain Raman emitting molecules adsorbed on such particles exhibit surface-enhanced Raman scattering due to the cou-

\footnotetext{
a)Electronic mail: c.ungureanu@utwente.nl.

b)Electronic mail: s.manohar@utwente.nl.
}

pling of the molecules' electronic states with the plasmon resonance band. ${ }^{3}$

Biomedical applications of GNRs based on these properties are emerging rapidly and include sensing, imaging, and therapy. The light scattering and emission properties of GNRs have promoted their use as excellent labels in studying biological and biochemical processes in cells using darkfield microscopy, ${ }^{4,5}$ Raman spectroscopy, ${ }^{3,6}$ optical coherence tomography, ${ }^{7,8}$ and multiphoton microscopy. ${ }^{9,10}$ Further, based on the wavelength dependence of the plasmon resonance on the refractive index of the local environment, GNRs have been shown to perform as biochemical sensors. ${ }^{11}$ Absorption of light by GNRs is followed by predominantly nonradiative de-excitation processes and the released heat and subsequent temperature rise have applications in improving photoacoustic signals. ${ }^{12,13}$ Further the temperature rise can be made sufficiently high to cause cell death by hyperthermia, which has potential for important therapeutic applications. ${ }^{14-16}$ In the last mentioned application, the ability to redshift LP peaks by tailoring the dimensions of the GNRs coupled with the fact that biological tissue is relatively transparent in the red and NIR wavelengths makes GNRs very attractive.

Modeling the optical properties of GNRs and their dependence on various particle and environmental conditions is of paramount importance in deciding applications and/or tailoring conditions to exploit performance optimally in these applications. Further, comparison of experimental data with simulated optical properties is a means of characterizing 
samples of nanoparticles in terms of the various parameters such as size, geometry, concentration, and dispersity that these properties are known to depend on. Modeling efforts in the recent past have concentrated on the discrete dipole approximation (DDA) method, ${ }^{17}$ the most popular realization of which is the DDSCAT code. ${ }^{18}$

Since the early studies by the group of Schatz and co-workers ${ }^{19}$ where the applicability of the DDA approach to simulate the extinction spectra of small metallic particles was shown, a variety of shapes and compositions have been studied including GNRs. ${ }^{20-23}$ Lee and El-Sayed ${ }^{24}$ used DDSCAT to show the relative contributions of scattering and absorption to the extinction as a function of aspect ratio and medium refractive index, as well as a preliminary study of the effect of end-cap shape. Further studies from the ElSayed group looked at the effect of effective particle size and composition. $^{25,26}$ Prescott and Mulvaney ${ }^{27}$ further investigated the effects of different capping geometries to the basic cylinder shape. They also showed the importance of using polydispersity in simulating extinction spectra. Kooij and Poelsema ${ }^{28}$ studied various morphologies to represent nanorods and showed the influence of electron surface scattering due to particle finite size effects.

While the KNOWLEDGEBASE is considerable in DDA applications for GNRs, there are inconsistencies in the choice of various parameters required for the simulations. In this article we call attention to this problem by showing the profound influence that these parameters have on the results. The effect of morphology, whether ellipsoid, rectangular, cylindrical, or hemispherically capped cylinder, has been studied in the past with observations that the one or the other gives a good agreement to the experiment. ${ }^{21,27,28}$ We show that this must be regarded with circumspection since in combination with a different source of the dielectric function of gold, fortuitous agreements may be found in certain cases. All in all, we show that there are several issues that should be considered before DDA results can be used to correctly interpret experimental data and to help characterize nanoparticles synthesized.

\section{MATERIALS AND METHODS}

\section{A. Gold nanospheres and nanorods}

Gold spheres of $25 \mathrm{~nm}$ were purchased from Aurion BV (Wageningen, The Netherlands) and spheres of $60 \mathrm{~nm}$ from British Biocell International (U.K.). GNRs were synthesized in the laboratory using the seed-mediated growth method of Nikoobakht and El-Sayed. ${ }^{29,30}$ In this wet-chemistry method, preformed gold spheres form the seed on which metal is grown along preferential directions directed by the surfactant cetyltrimethylammonium bromide (CTAB) in the presence of silver nitrate $\left(\mathrm{AgNO}_{3}\right)$. Excellent tuning of aspect ratios is achieved by changing the volumes of $\mathrm{AgNO}_{3}$ in the growth solution. Details may be found in Ref. 30 but we summarize the protocol here. Gold spheres as seed were prepared by reducing $5 \mathrm{ml} 0.0005 \mathrm{M}$ tetrachloroauric acid with $0.6 \mathrm{ml}$ $0.01 \mathrm{M}$ sodium borohydride in the presence of $5 \mathrm{ml} 0.2 \mathrm{M}$ CTAB. Within $15 \mathrm{~min}, 0.014 \mathrm{ml}$ of this seed solution was added to growth solutions containing $5 \mathrm{ml} 0.0005 \mathrm{M}$ tetra- chloroauric acid, $5 \mathrm{ml} \mathrm{0.2} \mathrm{M} \mathrm{CTAB,} 0.07 \mathrm{ml} \mathrm{0.1} \mathrm{M} \mathrm{ascorbic}$ acid, and $[0.05,0.1,0.2,0.25] \mathrm{ml}$ of $0.006 \mathrm{M} \mathrm{AgNO}_{3}$.

Sizes and shapes of the nanoparticles were examined in high resolution scanning electron microcopy (HR-SEM) digital images; dimensions were measured from at least 250 particles using the NI-Vision module (Labview, National Instruments). Extinction spectra of the nanoparticles were measured using the Shimadzu PC3101 UV-visible-NIR spectrophotometer.

\section{B. The DDSCAT package}

DDSCAT 6.1 (Ref. 18) is a FORTRAN package that implements the DDA method to simulate interaction of electromagnetic radiation with particles of arbitrary shape and composition. The method is described in details elsewhere. ${ }^{20-23,31}$ Briefly, the particle is subdivided into $N$ polarizable points located on a cubic lattice with an interdipole distance $d$ given by $V=N d^{3}$, where $V$ is the volume of the particle. ${ }^{18}$ The radiation scattered and absorbed by the target is computed taking into consideration dipole-dipole interactions. A large variety of particle shapes may be studied where the size of the particle is represented by the effective radius $r_{\text {eff }}$ $=(3 V / 4 \pi)^{1 / 3}$, which is the radius of a sphere having a volume equal to that of the particle. The output parameters from the simulation are extinction, absorption, and scattering efficiencies $\left(Q_{\text {ext }}, Q_{\text {abs }}, Q_{\text {sca }}\right)$, which yield the corresponding cross sections of the particle when multiplied by $\pi r_{\mathrm{eff}}^{2}$. In all simulations, the results are the average of the two cases of incident light polarization perpendicular to nanorod transverse and longitudinal axes, respectively.

For all simulations, the number of dipoles $(N)$ used to discretize the particle was chosen to satisfy the accuracy criterion $^{18}$

$$
|m| k d<0.5,
$$

where $m$ is the complex refractive index of the target material, $k=2 \pi / \lambda$ is the angular wave number with $\lambda$ as the wavelength of light, $d$ is the interdipole distance, and $V$ is the volume of the particle. All DDSCAT simulations were run on the Netherlands National Computer cluster (LISA).

\section{Choice of nanorod shape in DDSCAT}

There have been prior studies to investigate the influence of morphology chosen to represent the nanorod shape on simulation results. Lee and El-Sayed ${ }^{24}$ and Prescott and Mulvaney ${ }^{27}$ showed that varying the end-cap shape from flat to hemispherical redshifts the wavelength of LP peak. While we use the hemispherically capped cylinder as approximating the nanorods we synthesize, we extend the work of Kooij and Poelsema ${ }^{28}$ and compare the simulations of ellipsoidal, cylindrical, rectangular, and hemispherically capped cylinder shape with experimental spectra. Henceforth, as in Ref. 24, we refer to the last shape as nanorod shape.

\section{Choice of dielectric function of the material in DDSCAT}

Information regarding the target composition is introduced via the complex dielectric function $\epsilon_{m}=\epsilon_{1}+i \epsilon_{2}$ or $m$ 

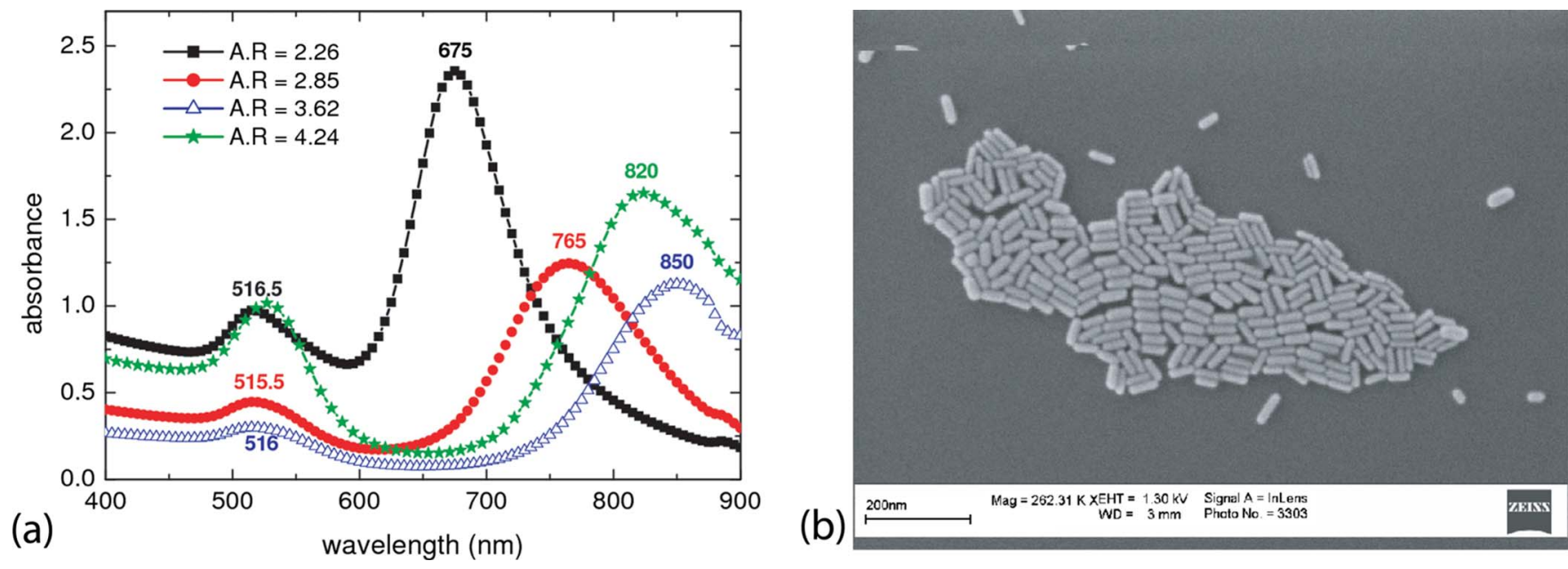

FIG. 1. (Color online) Absorbance spectra of GNRs with aspect ratios of 2.26, 2.85, 3.62, and 4.24. (b) HR-SEM image of particles with aspect ratio of 2.85.

$=n+i k$. Prior reports have been used without justification experimental data tabulated by either Johnson and Christy, ${ }^{32}$ Palik, ${ }^{33}$ or Weaver et al. ${ }^{34}$ Further, sometimes bulk values and at other times size correction modifications to account for surface damping have been used. References 20, 22, 23, 26 , and 35 extracted the bulk values from Johnson and Christy; ${ }^{32}$ Refs. 21, 24, 25, and 28 used bulk values from Palik; ${ }^{33}$ Ref. 27 used the size-corrected values from Weaver. ${ }^{34}$ In this work we compare the results using dielectric functions from all the three sources, both bulk and surface corrected. Irregularities in the data of Palik ${ }^{33}$ were removed to obtain a smooth variation in dielectric function in the values. ${ }^{28}$ Size correction was performed according to the standard manner ${ }^{20}$ by including an additional damping term to account for the collision of conduction electrons with the particle surface. This is expressed as

$$
\epsilon_{m}=\epsilon_{b}+\Delta \epsilon,
$$

where

$$
\Delta \epsilon=\frac{\omega_{p}^{2}}{\omega(\omega+i / \tau)}-\frac{\omega_{p}^{2}}{\omega\left(\omega+i / \tau+i / \tau_{a}\right)},
$$

where $\epsilon_{b}$ is the experimental bulk metal value of the dielectric function, $\omega_{p}$ is the plasma angular frequency, $1 / \tau$ is the damping constant, and $1 / \tau_{a}$ is the surface damping term given by $\nu_{f} / r_{\text {eff }}$ with $\nu_{f}$ as the Fermi velocity. For size correction, an effective radius of $12 \mathrm{~nm}$ was used, which is the average effective radius for GNRs synthesized in our laboratory.

\section{EXPERIMENTAL AND NUMERICAL RESULTS}

\section{A. Gold nanorod synthesis}

The synthesis protocol yielded GNRs with LP resonant peaks designed to occupy wavelengths in the region of 675$850 \mathrm{~nm}$ by changing the $\mathrm{AgNO}_{3}$ volume in the growth solution. ${ }^{30}$ Experimental absorbance spectra of four sets of GNRs with aspect ratios of 2.26, 2.85, 3.62, and 4.24 are shown in Fig. 1(a). The positions of the LP peaks due to conduction electron oscillations along the long axes of the particles are seen to redshift with increasing lengths. The transverse plasmon peaks due to oscillations along the short axes remain steady in the region of $516 \mathrm{~nm}$. Figure 1(b) is a HR-SEM image of a typical selection of GNRs with aspect ratio of 2.85. The shapes of the nanorods appear to be hemispherically capped cylinders. Information extracted from the HR-SEM images regarding mean values of length, width, aspect ratio, and position of the LP peak are presented in Table I.

\section{B. Shape}

Figures 2(a)-2(d) show the simulated extinction spectra for GNRs with aspect ratios of 2.26, 2.85, 3.62, and 4.24 when the particle morphology is treated as ellipsoidal, rectangular, cylindrical, and nanorod shaped. The positions of the LP maximum from spectrophotometric data of the respective sols are also shown in the graphs with dotted lines. Dipole numbers of 35000 and a refractive index of 1.33 for the environment were chosen. For dielectric function, sizecorrected values from Johnson and Christy ${ }^{32}$ were chosen due to their extensive usage in the gold/silver nanotechnology community.

There is considerable variation in position, amplitude, and width of the resonance maxima with the different shapes. $^{24,27,28}$ The order of occurrence in the wavelength of the plasmon peaks of various shapes for the same size parameters is in agreement with earlier reports. ${ }^{24,27,28}$ The position of the plasmon peak calculated using the realistic nanorod shape has a poor match with the experimental value, being blueshifted by more than $50 \mathrm{~nm}$ in three cases and redshifted by $30 \mathrm{~nm}$ in one case. The cylindrical shape ap-

TABLE I. Mean values of size-related parameters for GNR samples.

\begin{tabular}{lcccc}
\hline \hline Batch & $\begin{array}{c}\text { LP peak position } \\
(\mathrm{nm})\end{array}$ & $\begin{array}{c}\text { Length } \\
(\mathrm{nm})\end{array}$ & $\begin{array}{c}\text { Width } \\
(\mathrm{nm})\end{array}$ & Aspect ratio \\
\hline I & 675 & $44.8 \pm 4.1$ & $19.8 \pm 2.9$ & $2.26 \pm 0.3$ \\
II & 765 & $45.1 \pm 5.5$ & $15.8 \pm 3.1$ & $2.85 \pm 0.6$ \\
III & 850 & $51.0 \pm 4.4$ & $14.1 \pm 2.1$ & $3.62 \pm 0.6$ \\
IV & 820 & $49.1 \pm 4.8$ & $11.5 \pm 1.5$ & $4.24 \pm 0.6$ \\
\hline \hline
\end{tabular}



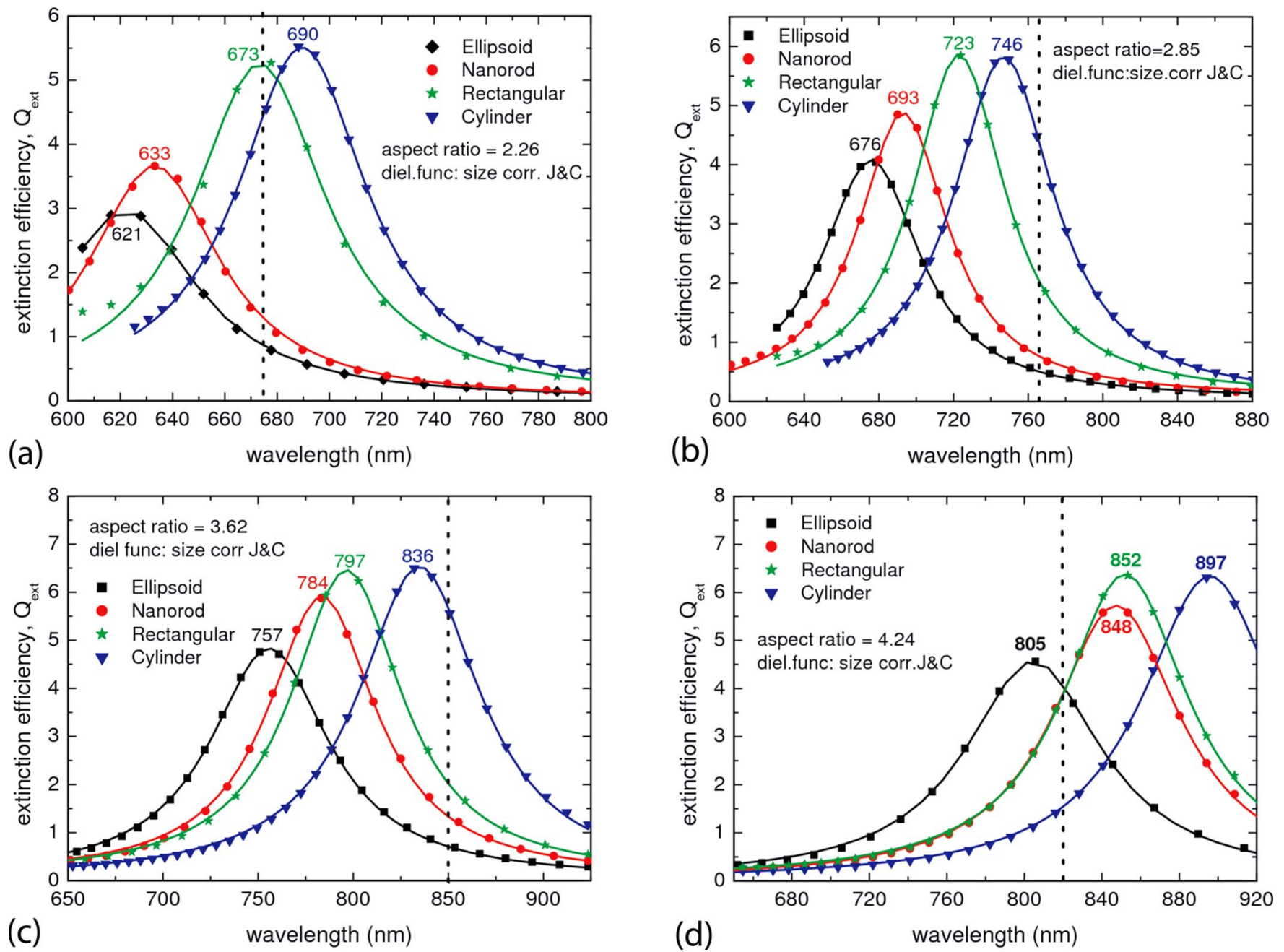

FIG. 2. (Color online) Calculated extinction spectra in the region of the LP wavelength for a GNR represented by different shapes for aspect ratios of (a) 2.26, (b) 2.85 , (c) 3.62 , and (d) 4.24 . The dotted line marks the experimental derived peak.

peared to be most favorable in three of the four cases; in one case there was a match using the rectangular shape.

\section{Dielectric function}

The real and imaginary parts $\left(\epsilon_{1}\right.$ and $\left.\epsilon_{2}\right)$ of the dielectric functions [see Eq. (2)] extracted from Johnson and Christy, ${ }^{32}$ Palik, ${ }^{33}$ and Weaver et al. ${ }^{34}$ are shown in Figs. 3(a) and 3(b), respectively. In the range of 500-600 nm, $\epsilon_{1}$ values from the three sources are closely similar. Values of $\epsilon_{2}$ from Johnson and Christy ${ }^{32}$ and Palik ${ }^{33}$ have an offset from each other in this range.

In the NIR regions, the data curves of $\epsilon_{1}$ from the three sources diverge. For $\epsilon_{2}$, the values from Johnson and Christy $^{32}$ and Palik ${ }^{33}$ maintain a similar trend with a small constant offset between each other; the $\epsilon_{2}$ from Weaver et $a l .{ }^{34}$ is lower and continuously diverges from other data curves.
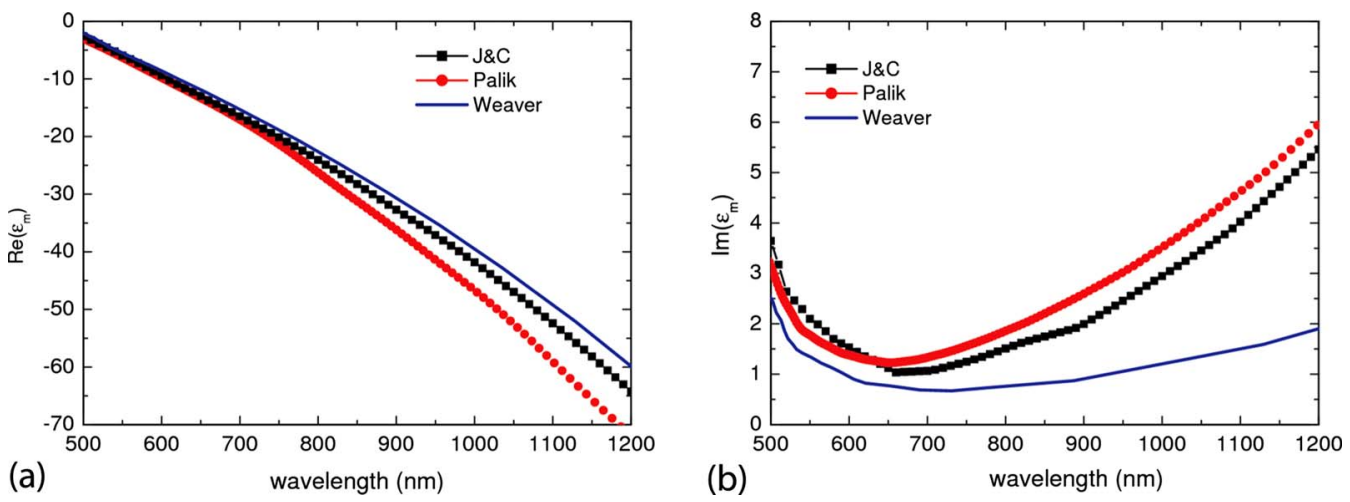

FIG. 3. (Color online) Bulk dielectric function of gold from Refs. 32-34. 

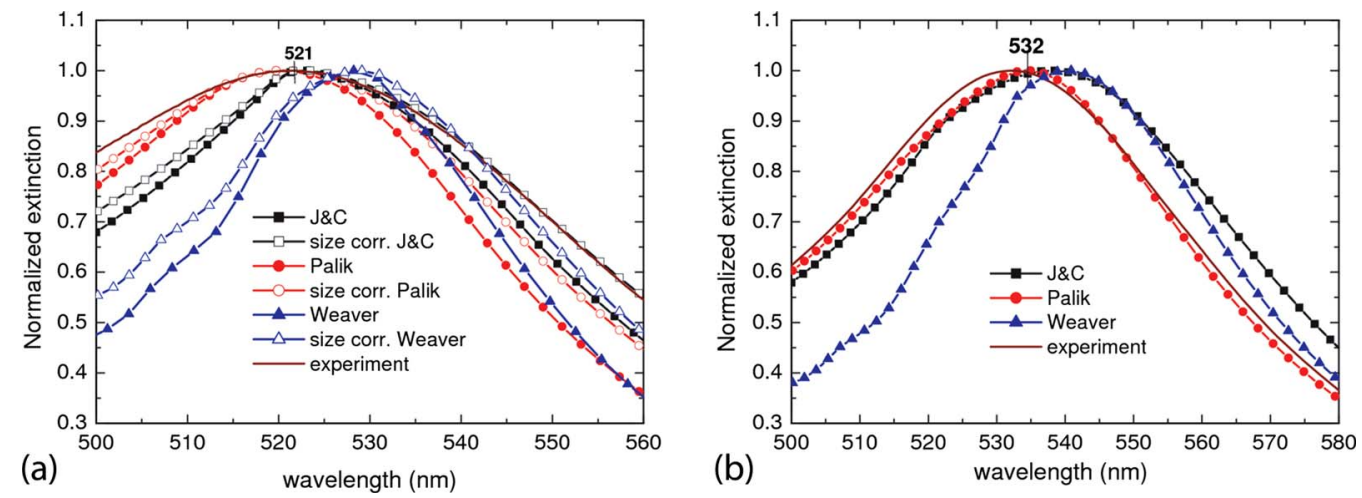

FIG. 4. (Color online) Comparison of simulated and experimental extinction spectrum of gold sphere: (a) 25 and (b) $60 \mathrm{~nm}$ diameter.

The normalized extinction efficiency spectra for gold spheres with diameters of 25 and $60 \mathrm{~nm}$ using bulk and size-corrected [see Eqs. (2) and (3)] dielectric function values from the three sources are shown in Figs. 4(a) and 4(b), respectively. The experimental extinction spectra of the respective sols are also plotted in the figures. Simulations were performed for 35000 dipoles and a local refractive index of 1.33 .

The most significant feature is that the position of the plasmon peak is predicted well within a few nanometers in all cases with a good match to experiment with the exception when data from Weaver et al. ${ }^{34}$ were used. This can be explained by the marginal difference between the data from Johnson and Christy ${ }^{32}$ and Palik ${ }^{33}$ in the region of the spectrum where the sphere's plasmon peak occurs. As expected, the size-corrected results while maintaining the resonance maximum position show broadening in the case of the smaller sphere but have hardly any difference for the larger sphere.

In sharp contrast to the situation with spheres, the nanorod simulations yield a wide range of plasmon peak positions using size-corrected dielectric functions from three sources. The reason for this lies in the growing differences between the dielectric functions from the three sources at longer wavelengths (Fig. 3). Further, none of the simulated peaks matches with the location of the experimental peaks.

The observation of Prescott and Mulvaney ${ }^{27}$ that using the data from Weaver et $a l^{34}$ with a cylindrical shape showed a good match between simulations and experiments was intriguing, and we decided to test the same with our experimental data. We combine the simulated extinction spectra for both nanorod and cylindrical shapes in one graph using size-corrected values of dielectric functions from the three sources. Figures 5(a)-5(d) show the results for aspect ratios of $2.26,2.85,3.62$, and 4.24 , respectively. All simulations were performed using 35000 dipoles and a local refractive index of 1.33 .

It is striking that there is a perfect match in two cases between simulations and experiment for the combination of data from Weaver et al. ${ }^{34}$ and cylindrical shape. In the case of particles with A.R 2.26 and for particles with A.R 4.24, a Palik-cylinder combination ${ }^{33}$ and a Palik-nanorod combination, ${ }^{33}$ respectively, give excellent matches to the experiment.
Amplitude differences between simulations using different combinations of shape and dielectric function are as high as $30 \%$.

\section{DISCUSSION}

We confirm the previous reports ${ }^{24,27,28}$ showing the strong dependence of shape on simulation results. Examination of HR-SEM images of the GNRs synthesized by us leads us to consider the hemispherically capped cylinder or nanorod shape as the most serious contender for appropriate morphology of the particles.

However, when this shape is used for simulation in combination with the widely accepted dielectric function of Johnson and Christy, ${ }^{32}$ a poor agreement with the experiment for the localization of the plasmon maximum is obtained. This unexpected outcome was then thought to result from an incorrect choice of the dielectric function.

This choice has little influence when gold nanospheres are simulated owing to the similarity of values from the three sources in the green region of the spectrum where the signature plasmon peak occurs. In contrast, there is a wide distribution of the spectra when nanorods are simulated depending on which dielectric function was chosen (Fig. 5). Prior work has ignored the large influence that the dielectric function has, choosing one or the other of the sources without justification and without recognizing the availability of other values.

We now revisit the shape issue but now acknowledge the effect of dielectric function. In two of the four cases, we obtain an agreement with the conclusion of Prescott and Mulvaney ${ }^{27}$ and obtain a perfect match with the experiment for a cylinder shape but under the qualification that dielectric functions of Weaver et al. ${ }^{34}$ were used. In two cases, (A.R. 2.26) a cylinder-Palik ${ }^{33}$ combination and (A.R. 4.24) a nanorod-Palik ${ }^{33}$ combination yielded an excellent agreement with the experiment.

It is obvious from electron microscopy that the particles we synthesize cannot be described by a cylindrical or rectangular shape but are closest to the nanorod shape. Assuming this as a known parameter, we look to appropriateness in dielectric function to explain the contradictory results. While it is difficult to judge the correctness of one source against the other, it is likely that all values obtained from thin films 


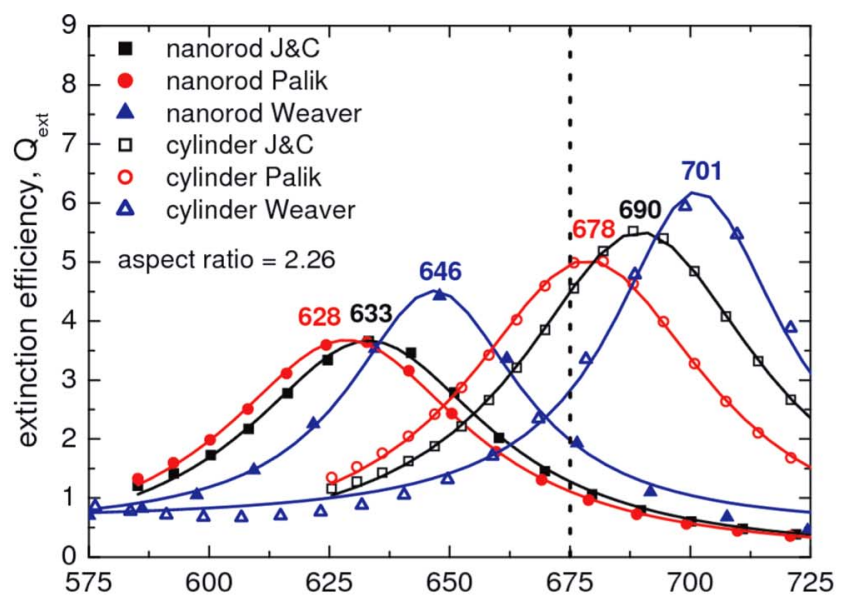

(a)

wavelength $(\mathrm{nm})$

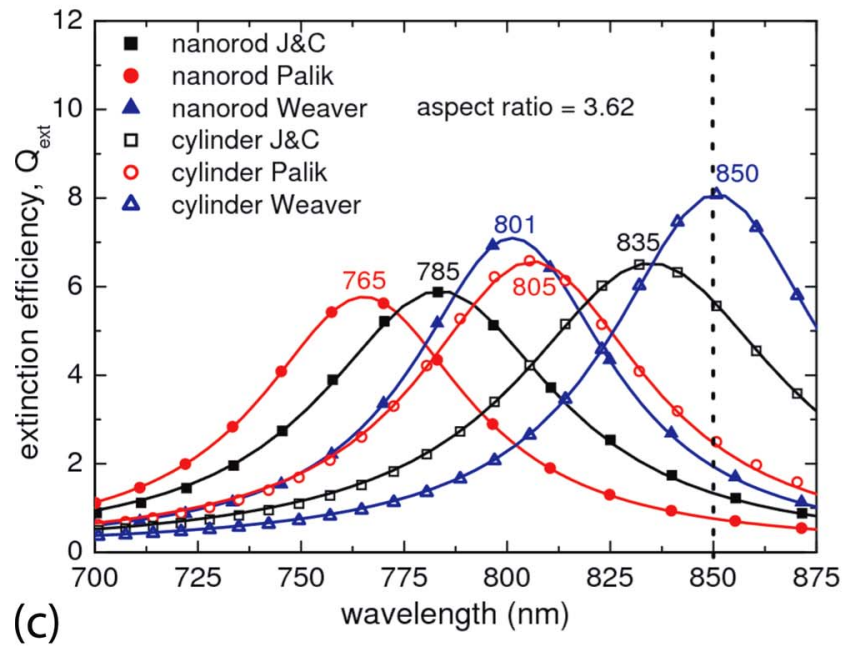

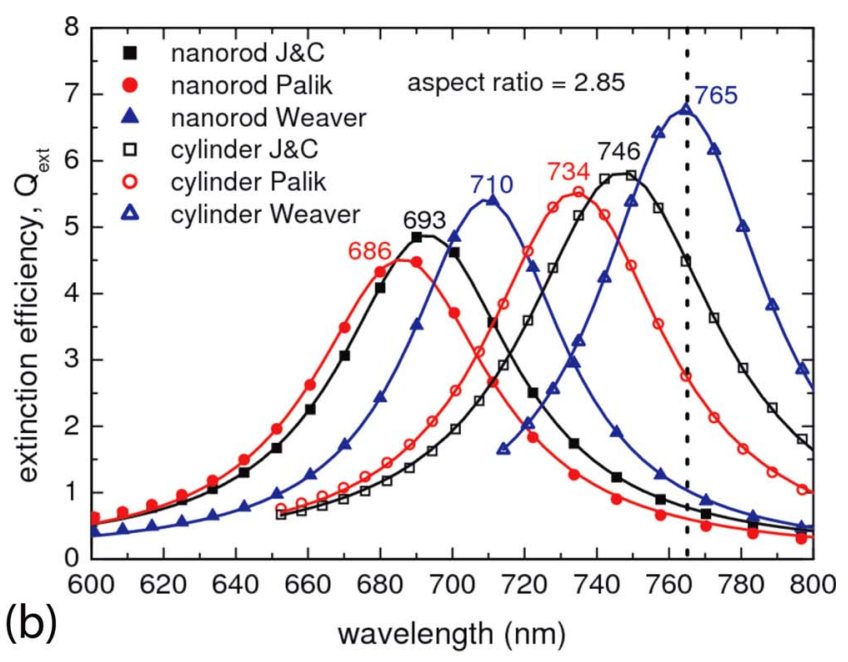

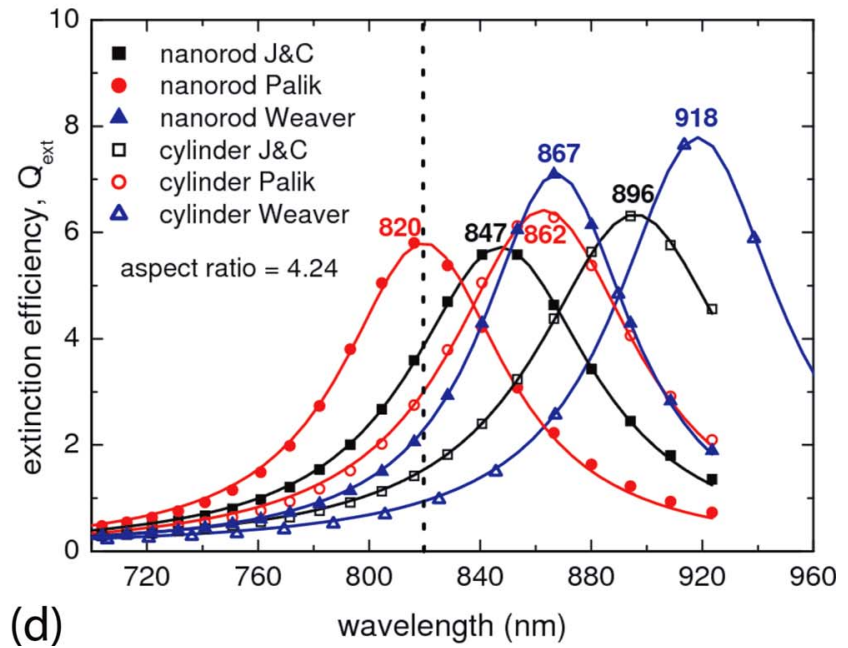

FIG. 5. (Color online) Simulated extinction spectrum using size-corrected dielectric functions and nanorods and cylinder shapes with aspect ratios of (a) 2.26, (b) 2.85 , (c) 3.62 , and (d) 4.24 .

and therefore bulk deviate from the dielectric constant of nanoparticulate matter. ${ }^{36}$

\section{CONCLUSIONS}

Locating the position of the resonance maxima of GNRs using DDSCAT simulations is not trivial. This position cannot be uniquely determined and depends upon the shape used to represent the particle and the source of dielectric function used. Further, the local environment and the sizing of the particles are important as well. The magnitude of the resonant peaks is also dependent on these factors but is less sensitive to the available choices compared with the position of peaks.

The examination of HR-SEM images points toward the use of hemispherically capped cylinders for the shape of the nanorods. However, it is not yet clear what dielectric function to use to obtain best fitting between simulations and experiment. In any case this work serves to draw attention to this drawback, which has not been recognized in earlier works.

The most important conclusion that we draw from our study is that with the present approach it is not possible to objectively compare experimental data with the simulations owing to various input parameters that can be used as tuning parameters to obtain an agreement. This drawback is best exemplified in Figs. 2(a) and 5(a) for the aspect ratio of 2.26, where an excellent agreement but fortuitous match to the experiment is obtained when the particle is modeled as rectangular and cylindrical, respectively, simply depending on the dielectric function chosen even when it is known that the shapes chosen are incorrect.

\section{ACKNOWLEDGMENTS}

This work is funded through the thrust area program NIMTIK of the University of Twente, the PRESMITT project (Grant No. IPD067771) of the SenterNovem program IOP Photonic Devices, and by the Nederlandse Wetenschappelijk Organisatie (NWO) and Stichting Technische Wetenschappen (STW) through Project No. TTF 6527. The use of supercomputer facilities was made available by the Stichting Nationale Computerfaciliteiten (National Computing Facilities Foundation, NCF) with financial support from the NWO.

${ }^{1}$ J. Pérez-Juste, I. Pastoriza-Santos, L. M. Liz-Marzan, and P. Mulvaney, Coord. Chem. Rev. 249, 1870 (2005).

${ }^{2}$ M. B. Mohamed, V. Volkov, S. Link, and M. A. El-Sayed, Chem. Phys. Lett. 317, 517 (2000).

${ }^{3}$ X. Huang, I. H. El-Sayed, W. Qian, and M. A. El-Sayed, Nano Lett. 7, 1591 (2007). 
${ }^{4}$ P. K. Jain, I. H. El-Sayed, and M. A. El-Sayed, Nanotoday 2, 18 (2007).

${ }^{5}$ H. Ding, K.-T. Yong, I. Roy, H. E. Pudavar, W. C. Law, E. J. Bergey, and P. N. Prasad, J. Phys. Chem. C 111, 12552 (2007).

${ }^{6}$ N. Félidj, G. Laurent, J. Grand, J. Aubard, G. Lévi, A. Hohenau, F. R. Aussenegg, and J. R. Krenn, Plasmonics 1, 35 (2006).

${ }^{7}$ A. L. Oldenburg, M. N. Hansen, D. A. Zweifel, A. Wei, and S. A. Bopart, Opt. Express 14, 6724 (2006).

${ }^{8}$ T. S. Troutman, J. K. Barton, and M. Romanowski, Opt. Lett. 32, 1438 (2007).

${ }^{9}$ H. Wang, T. B. Huff, D. A. Zweifel, W. He, P. S. Low, A. Wei, and J.-X. Cheng, Proc. Natl. Acad. Sci. U.S.A. 102, 15752 (2005).

${ }^{10}$ N. J. Durr, T. Larson, D. K. Smith, B. A. Korgel, K. Sokolov, and A. Ben-Yakar, Nano Lett. 7, 941 (2007).

${ }^{11}$ C.-D. Chen, S.-F. Cheng, L.-K. Chau, and C. R. C. Wang, Biosens. Bioelectron. 22, 926 (2007).

${ }^{12}$ K. Kim, S. W. Huang, S. Ashkenazi, M. O'Donnell, A. Agarwal, N. A. Kotov, M. F. Denny, and M. J. Kaplan, Appl. Phys. Lett. 90, 223901 (2007).

${ }^{13}$ M. Eghtedari, A. A. Oraevsky, J. A. Copland, N. A. Kotov, A. Conjusteau, and M. Motamedi, Nano Lett. 7, 1914 (2007).

${ }^{14}$ C. H. Chou, Cheng. D. Chen, and C. R. Chris Wang, J. Phys. Chem. B 109, 11135 (2005).

${ }^{15}$ T. B. Huff, L. Tong, Y. Zhao, M. N. Hansen, J.-X. Cheng, and A. Wei, Nanomedicine 2, 125 (2007).

${ }^{16}$ L. Tong, Y. Zhao, T. B. Huff, M. N. Hansen, A. Wei, and J.-X. Cheng, Adv. Mater. (Weinheim, Ger.) 19, 3136 (2007).

${ }^{17}$ E. M. Purcell and C. R. Pennypacker, Astrophys. J. 186, 705 (1973).

${ }^{18}$ B. T. Draine and P. J. Flatau, User Guide for the Discrete Dipole Approximation Code DDSCAT.6.1, http://arxiv.org/abs/astro-ph/xxx.

${ }^{19}$ W.-H. Yang, G. C. Schatz, and R. R. van Duyne, J. Chem. Phys. 103, 869
(1995).

${ }^{20}$ N. Félidj, J. Aubard, and G. Lévi, J. Chem. Phys. 111, 1195 (1999).

${ }^{21}$ A. Brioude, X. C. Jiang, and M. P. Pileni, J. Phys. Chem. B 109, 13138 (2005).

${ }^{22}$ G. Yin, S.-Y. Wang, M. Xu, and L.-Y. Chen, J. Korean Phys. Soc. 49, 2108 (2006).

${ }^{23}$ A. L. Gonzalez and C. Noguez, J. Comput. Theor. Nanosci. 4, 231 (2007).

${ }^{24}$ K. S. Lee and M. A. El-Sayed, J. Phys. Chem. B 109, 20331 (2005).

${ }^{25}$ K. S. Lee and M. A. El-Sayed, J. Phys. Chem. B 110, 19220 (2006).

${ }^{26}$ P. K. Jain, K. S. Lee, I. H. El-Sayed, and M. A. El-Sayed, J. Phys. Chem. B 110, 7238 (2006).

${ }^{27}$ S. W. Prescott and P. Mulvaney, J. Appl. Phys. 99, 123504 (2006).

${ }^{28}$ E. Stefan Kooij and B. Poelsema, Phys. Chem. Chem. Phys. 8, 3349 (2006).

${ }^{29}$ B. Nikoobakht and M. A. El-Sayed, Chem. Mater. 15, 1957 (2003).

${ }^{30}$ R. Rayavarapu, W. Peteresen, C. Ungureanu, T. G. van Leeuwen, and S. Manohar, Int. J. Biomed. Imaging 29817, 29817-1 (2007).

${ }^{31}$ B. T. Draine and P. J. Flatau, J. Opt. Soc. Am. A Opt. Image Sci. Vis 11, 1491 (1994).

${ }^{32}$ P. B. Johnson and R. W. Christy, Phys. Rev. B 6, 4370 (1972).

${ }^{33}$ E. D. Palik, Handbook of Optical Constants of Solids (Academic, New York, 1985).

${ }^{34}$ J. Weaver, C. Krafka, D. Lynch, and E. Koch, Physics Data: Optical Properties of Metals, Part 2: Noble Metals, Aluminium, Scandium, Yttrium, the Lanthanides and the Actinides (Fach-Informations Zentrum, Karlsruhe, 1981).

${ }^{35}$ C. Ungureanu, R. G. Raja, T. G. van Leeuwen, and S. Manohar, Proc. SPIE 6631, 663108 (2007).

${ }^{36}$ P. Stoller, V. Jacobsen, and V. Sandoghdar, Opt. Lett. 31, 2474 (2006). 